\title{
Smoking cessation and carotid atherosclerosis: the Guangzhou Biobank Cohort Study-CVD
}

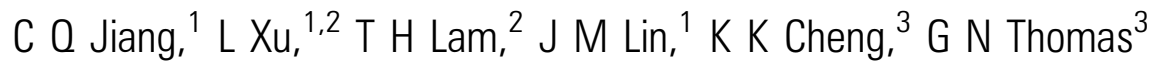

\begin{abstract}
${ }^{1}$ Molecular Epidemiology Research Centre, Guangzhou Number 12 Hospital, Guangzhou, China

${ }^{2}$ Department of Community Medicine, School of Public Health, University of Hong Kong, Hong Kong SAR, China ${ }^{3}$ Department of Public Health and Epidemiology, University of Birmingham, Birmingham, UK

\section{Correspondence to} Professor Tai Hing Lam, Department of Community Medicine, School of Public Health, The University of Hong Kong, Hong Kong, China; hrmrlth@hkucc.hku.hk
\end{abstract}

Accepted 23 September 2009 Published Online First 12 October 2009

\begin{abstract}
Introduction Smoking has been shown to be associated with carotid atherosclerosis in cross-sectional and prospective studies in Western populations. However, few studies have examined the reversal of risk resulting from quitting smoking, and the results are conflicting. Methods 959 men aged 50-85 years were randomly selected from phase III (2006-2007) of the Guangzhou Biobank Cohort Study into this cross-sectional study. Common carotid artery intima-media thickness (CCAIMT) was measured by B-mode ultrasonography, and carotid artery plaques were identified. Major cardiovascular risk factors, including fasting triglyceride, low-density and high-density lipoprotein (LDL and HDL) cholesterol and glucose, and systolic and diastolic blood pressure, were assessed.
\end{abstract}

Results CCA-IMT and the number of carotid plaque increased from never to former to current smokers (both $p \leq 0.001)$. Among former smokers compared to current smokers, after adjustment for cigarette pack-years and other potential confounders, the adjusted ORs $(95 \% \mathrm{CI})$ for quitting for $1-9,10-19$ and $20+$ years were 0.77 (0.47 to 1.26$), 0.45$ (0.26 to 0.79 ) and 0.37 (0.17 to $0.77)$ for the presence of CCA atherosclerosis, and 0.69 (0.43 to 1.12$), 0.47$ (0.27 to 0.82 ) and 0.45 (0.23 to 0.96 ) for the presence of carotid plaques, respectively. Longer duration of quitting smoking was also significantly associated with decreasing risk of the severity of CCA atherosclerosis and carotid plaques (all $p \leq 0.001$ ).

Conclusion Smoking cessation was beneficial in attenuating the risk of carotid atherosclerosis associated with cigarette smoking. The short duration of cessation in earlier studies is a likely explanation for the inconsistent results.

\section{INTRODUCTION}

China is the world's largest tobacco producer and consumer. Data from the 1996 National Prevalence Survey showed that about $30 \%$ of the world's cigarettes were consumed by China's 350 million smokers. ${ }^{1}$ In 2002, the prevalence of current smoking was $66 \%$ in men and $3.1 \%$ in women aged 15 years or older. ${ }^{2}$ Data from phase I (2003-2004) of the Guangzhou Biobank Cohort Study (GBCS) showed that the prevalence of ever smokers in older Chinese people aged 50 years or above was $57.7 \%$ and $5.3 \%$ in men and women, respectively. ${ }^{3}$ Likewise, China has more attributable tobacco-related deaths than any other country. There remains comparatively low awareness of the smoking-related risk of cardiovascular disease among healthcare professionals and the public. Studies of the health effects, including the beneficial effects associated with quitting, within Chinese populations remain limited, yet such specific data should be of great importance in increasing awareness and changing population smoking habits.

Carotid artery plaques and intima-media thickness (IMT), as measured by B-mode ultrasonography, are non-invasive quantitative measures of the presence and severity of carotid atherosclerosis. ${ }^{4}$ Carotid IMT has been associated with cardiovascular risk factors as well as cardiovascular events in cumulative studies, and has been wellaccepted as a marker of subclinical atherosclerosis. ${ }^{5-14}$ However, the results are not very robust as most of these studies were in hospitalized patients and the effect of underlying diseases on IMT finding was not examined. Methodological differences in the details of ultrasound examinations, such as the use of maximal or mean IMT or the measurement of near or far wall IMT, could affect the results. Furthermore, some studies have small sample size, which resulted in imprecise estimates of the risk.

Cross-sectionally, carotid atherosclerosis (thicker IMT or plaques) is associated with major cardiovascular risk factors such as total cholesterol, lowdensity lipoprotein (LDL) cholesterol, fasting plasma glucose, systolic and diastolic blood pressure, and inversely with high-density lipoprotein (HDL) cholesterol. ${ }^{9-11}$ Prospectively, these surrogate markers predict increased risk of cardiovascular disease, such as myocardial infarction, coronary heart disease and stroke. ${ }^{12-14}$

Smoking has been shown to be associated with carotid atherosclerosis in cross-sectional ${ }^{4} 1516$ and prospective studies ${ }^{17} 18$ in Western populations. However, few studies have examined the reversal of risk resulting from quitting smoking, and the results are conflicting. ${ }^{17} 1920$ Similarly, only two cross-sectional studies describing Chinese subjects can be found in the literature. A study from Taiwan found that age, systolic blood pressure and fasting blood glucose were independent risk factors for carotid IMT and plaques, but smoking was not. ${ }^{21}$ Thomas et al ${ }^{11}$ included Chinese from Hong Kong, Macau, San Francisco and China, and found that smoking was associated with adverse levels of the vascular disease surrogate markers, IMT and endothelial function. Neither of the above-mentioned studies examined risk reversal from quitting. Interventional studies showed that smoking cessation, as one lifestyle modification, can slow the progression of coronary atherosclerosis. ${ }^{22-25}$ However, whether lifestyle intervention such as smoking cessation can reverse the progression of carotid atherosclerosis is still controversial. ${ }^{26}{ }^{27}$

We present here the first report in a Chinese population exploring the dose-response relationships 
between the presence and severity of common carotid artery (CCA) atherosclerosis and plaques and smoking and quitting.

\section{MATERIALS AND METHODS}

The GBCS is a three-way collaboration among Guangzhou Number 12 People's Hospital, China, University of Hong Kong and University of Birmingham, UK. The study aims to examine environmental and occupational factors, and genetic and lifestyle determinants of common chronic diseases. A community social and welfare association, "The Guangzhou Health and Happiness Association for the Respectable Elders," aligned with the municipal government was chosen as a sampling frame. It is a large organisation with branches throughout Guangzhou, and its membership is open to anyone for a nominal discretionary fee of 48 Yuan (US\$6) per year. It has a citywide network with around 107000 members, 95\% of whom are permanent residents, approximately $7 \%$ of the Guangzhou population of that age strata. During 2003-2007, we have recruited about 30000 older subjects aged 50 years or above from Guangzhou in southern China. Details of participant recruitment and methods in the GBCS have been reported previously. ${ }^{28}$

In a more detailed substudy on cardiovascular disease and diabetes, 1996 subjects were randomly selected from a list of eligible subjects during the phase III of GBCS (November 2006-December 2007). ${ }^{29}$ A standardized questionnaire was used to assess occupational exposure; family and personal disease history; cognitive function and lifestyle, including smoking, drinking status and physical activity (International Physical Activity Questionnaire). ${ }^{30}$

Resting seated blood pressure was measured three times using an automated sphygmomanometer (Omron 705CP) that had been previously validated by comparison with the manual mercury sphygmomanometer, ${ }^{31}$ and the last two measurements were averaged for analysis. Height and weight were measured using a standardized procedure that had been described elsewhere. ${ }^{29}$ Fasting parameters including plasma glucose and lipids (triglyceride, total, LDL, and HDL cholesterol) were measured in the Clinical Laboratory of the Guangzhou Number 12 Hospital using standardized procedures.

Of the 992 men, 959 had a carotid B-mode colour ultrasonographic examination using ALT HDI 3000 mainframe with a high-resolution, linear array scanner (medium frequency 7.5 MHz) performed by a specialist physician. The operators were registered ultrasound doctors who had a professional certificate for colour Doppler ultrasound measurement awarded by the Ministry of Health of China. All scans were performed following a predetermined, standardized scanning protocol for the right and left carotid arteries using images of the far wall of the distal $10 \mathrm{~mm}$ of the common carotid arteries. Three scanning angles, with the image focused on the posterior wall, were recorded from the angle showing the greatest distance between the lumen-intima interface and the media-adventitia interface. Carotid IMT measurements were performed off-line with the use of automated image analysis software. All scans were analysed by the same physician, blinded to subjects' information. When thicker IMT or carotid plaque was observed, at least two physicians, including one chief physician, discussed and made the final decision. Details on the procedure used in this study and its reproducibility have been published elsewhere. ${ }^{29}$

The presence of CCA atherosclerosis was diagnosed if one or both of the left or right CCA-IMT was $\geq 1.0 \mathrm{~mm}$ or with a diameter stenosis $\geq 20 \%$. ${ }^{14} 32$ The severity of the CCA-IMT was graded into three categories (grade 0 for CCA-IMT $<1.0$ $\mathrm{mm}$ or no observable CCA plaque; grade 1 for CCA-IMT from
1.0 to $1.2 \mathrm{~mm}$; grade 2 for CCA-IMT $>1.2 \mathrm{~mm}$ or with a diameter stenosis $\geq 20 \%$ ). The presence of a plaque was defined as a distinct area protruding $\geq 1.2 \mathrm{~mm}$ into the vascular lumen of the carotid artery. ${ }^{33}$ Participants with one or more plaques in the CCA, internal carotid arteries or bifurcations of carotid arteries, on the right or left side were categorized as having carotid plaques. The severity of carotid plaque was assessed by the total number of plaques located in carotid and classified as none, one, two or more plaques.

Well-trained interviewers collected the smoking history using a standardized questionnaire. Smoking was defined as having smoked at least one cigarette per day or seven cigarettes per week for at least half a year. "Current smoker" was defined by answering "yes" to the question, "Do you smoke cigarettes now?" "Former smoker" was defined as "used to smoke but not smoking currently". Information on duration of quitting smoking (years) was collected. The reliability of the questionnaire was tested in 200 subjects with $\kappa$ values of 0.88 and 0.96 for the two questions about smoking status, respectively. ${ }^{3}$

The study was funded by an NSFC/RGC (No 30518001; HKU720/05) grant and received ethical approval from the Guangzhou Medical Ethics Committee of the Chinese Medical Association, Guangzhou, China. All participants gave written, informed consent before participating in the study.

All data analysis was conducted using Stata/SE 8.0. Statistical significance was set at a two-tailed $p<0.05 . \chi^{2}$ test and analysis of variance were used to assess the association between smoking status and categorical variables and continuous variables, respectively, and analysis of covariance was used for the adjustment of covariates. Binary logistic regression models were used to calculate ORs of presence of carotid plaques and CCA atherosclerosis without and then with adjustment for multiple cardiovascular risk factors. The $p$ value for trend was derived by linear regression models. Potential confounders assessed included age (years), body mass index $\left(\mathrm{kg} / \mathrm{m}^{2}\right)$, education (primary or below, middle school, college or above), physical activity (physically active, moderate and inactive), ${ }^{30}$ blood pressure (systolic and diastolic, $\mathrm{mm} \mathrm{Hg}$ ), fasting plasma glucose ( $\mathrm{mmol} / \mathrm{l})$, total cholesterol (mmol/l), HDL cholesterol ( $\mathrm{mmol} / \mathrm{l})$, LDL cholesterol $(\mathrm{mmol} / \mathrm{l})$ and triglyceride $(\mathrm{mmol} / \mathrm{l})$. Associations with discrete ordinal variables were tested by using the OLOGIT procedure, which performed multivariate logistic regression allowing for linear modelling of an ordinal dependent variable having more than two levels. This method was chosen because of the ordinal natural of the severity of carotid plaque and CCA atherosclerosis.

\section{RESULTS}

Of the 959 subjects, $25.7 \%$ (95\% CI $22.9 \%$ to $28.5 \%$ ) were former smokers and $35.2 \%$ (95\% CI $32.2 \%$ to $38.4 \%$ ) were current smokers. Among the former smokers, the median (interquartile range) pack-years for those quitting for 1-9, $10-19$ and $20+$ years were 32 (18-45), 25 (14-39) and 14 (6-26), respectively; $41.3 \%(38.2-44.5 \%)$ had CCA atherosclerosis and $33.8 \%(30.8 \%-36.9 \%)$ had at least one carotid plaque. Table 1 shows that the mean (SD) age increased from current smokers to never smokers to former smokers $(p<0.001)$. Compared to never smokers, current smokers and former smokers tended to have a lower education level and be less physically active. Table 2 shows that the mean of the left and right CCA-IMT and the number of carotid plaques increased from never to former to current smokers (all $\mathrm{p} \leq 0.001$ ). After adjusting for age, education, physical activity, body mass index, systolic and diastolic blood pressure, the mean CCA-IMT increased from $726 \mu \mathrm{m}$ in the never smokers to $767 \mu \mathrm{m}$ in the 
Table 1 Demographic characteristics of 959 older Chinese men

\begin{tabular}{|c|c|c|c|}
\hline & \multicolumn{3}{|c|}{ Smoking status } \\
\hline & $\begin{array}{l}\text { Never } \\
(\mathrm{n}=375)\end{array}$ & $\begin{array}{l}\text { Former } \\
(n=246)\end{array}$ & $\begin{array}{l}\text { Current } \\
(n=338)\end{array}$ \\
\hline Age, mean (SD), years & $62.1(6.8)$ & $63.6(7.0) \dagger$ & $60.8(6.3) \neq 9$ \\
\hline \multicolumn{4}{|l|}{ Education, n (\%) } \\
\hline Primary or below & $62(16.5)$ & $73(29.7)$ & $106(31.4)$ \\
\hline Middle school & $216(57.6)$ & $145(58.9)$ & $195(57.7)$ \\
\hline College or above & $97(25.9)$ & $28(11.4)$ & $37(10.9) \S$ \\
\hline \multicolumn{4}{|l|}{ Physical activity, n (\%)* } \\
\hline Active & $205(55.7)$ & $147(59.8)$ & $154(48.4)$ \\
\hline Moderate & $134(36.4)$ & $77(31.3)$ & $121(38.1)$ \\
\hline Inactive & $29(7.9)$ & $22(8.9)$ & $43(13.5)^{* *}$ \\
\hline BMI, mean (SD), kg/m² & $24.0(2.9)$ & $23.9(2.9)$ & $23.1(3.1) \neq 9$ \\
\hline $\mathrm{TG}$, mean (SD), mmol/l & $1.91(1.6)$ & $1.95(1.4)$ & $1.93(1.7)$ \\
\hline $\mathrm{HDL}$, mean (SD), mmol/l & $1.45(0.4)$ & $1.47(0.3)$ & $1.43(0.4)$ \\
\hline LDL, mean (SD), mmol/l & $3.20(0.59)$ & $3.30(0.64)$ & $3.20(0.64)$ \\
\hline Total cholesterol, mean (SD), mmol/l & $5.55(0.99)$ & $5.72(1.03)$ & $5.57(1.06)$ \\
\hline $\mathrm{SBP}$, mean (SD), mm Hg & $132.1(19.9)$ & $132.5(19.5)$ & $126.9(20.5) \neq 9$ \\
\hline $\mathrm{DBP}$, mean (SD), mm Hg & $76.7(10.7)$ & $76.2(9.9)$ & $74.3(10.8) 9$ \\
\hline $\mathrm{FPG}$, mean (SD), $\mathrm{mmol} / \mathrm{l}$ & $5.64(1.1)$ & $5.64(1.2)$ & $5.57(1.7)$ \\
\hline
\end{tabular}

*Participants with missing data were not included here only.

$\dagger p<0.05$ versus never smoker.

$\neq \mathrm{p}<0.01$ versus former smoker.

$\S p<0.01$ for $\chi^{2}$ test.

$\llbracket \mathrm{p}<0.01$ versus never smoker

${ }^{* *} \mathrm{p}<0.05$ for $\chi^{2}$ test.

$\mathrm{BMI}$, body mass index; TG, triglyceride; HDL, high-density lipoprotein cholesterol; LDL, lowdensity lipoprotein cholesterol; SBP, systolic blood pressure; DBP, diastolic blood pressure; $\mathrm{FPG}$, fasting plasma glucose.

former smokers, and 781 and $775 \mu \mathrm{m}$ in the current smokers with a pack-years of $1-39$ and $40+$, respectively $(p<0.001$; table 2). Table 3 shows that the presence and severity of CCA atherosclerosis and carotid plaques were associated with smoking status. Significant trends were observed from never smokers to former smokers to current smokers. Significant trends were also found for number of cigarettes smoked per day and years of smoking and pack-years of smoking, with the latter showing the greatest gradient (data not shown). The adjusted ORs $(95 \% \mathrm{CI})$ for the presence of CCA atherosclerosis and carotid plaque in current smokers (pack-years $40+$ ) was 2.47 (1.44 to 4.23 ) and 2.43 (1.45 to 4.05), respectively (all $p$ for trend $<0.001$ ). Analysis using ordinal logistic regression (model B) showed similar results.
Table 4 shows that, for former smokers compared to current smokers, there were decreasing trends of the adjusted ORs by increasing years of quitting ( $p \leq 0.001$ for both the presence and severity of CCA atherosclerosis and carotid plaque). After adjusting for pack-years (model A III), the beneficial effects of quitting smoking remained unchanged, and the adjusted ORs (95\% CI) for quitting for $1-9,10-19$ and $20+$ years were 0.77 (0.47 to 1.26$), 0.45$ (0.26 to 0.79 ) and 0.37 (0.17 to 0.77 ), respectively ( $p$ for trend $<0.001$ ) for the presence of CCA atherosclerosis, and 0.69 (0.43 to 1.12$), 0.47(0.27$ to 0.82$)$ and 0.45 (0.23 to 0.96 ), respectively ( $p$ for trend 0.002 ) for the presence of carotid plaques. Those who had quit for 20 years or more had a one-half to two-thirds reduction in risk. Ordinal logistic regression (model B I and B II), with or without adjustment for pack-years smoked, showed similar results.

\section{DISCUSSION}

Our study shows a reduction of risk for carotid atherosclerosis among former smokers, with the risk decreasing with increasing duration of smoking cessation, after adjusting for many potential confounders. We were also able to demonstrate the reduction of risks resulting from quitting, after adjustment of pack-years of cigarette smoke exposure. We confined our results to men, as few Chinese women smoke. ${ }^{28} 34$ Although the levels of most of the measured cardiovascular disease risk factors in the former smokers resembled those in never smokers, which may have contributed to the observed improvements in CCA-IMT, a number of those parameters were significantly worse than those in the smokers, namely body mass index and blood pressure, which would have offset some of the benefits of quitting smoking. To address potential confounding of these concomitant changes, these parameters were adjusted as covariates in the analyses. Given data from Western populations, our results, although cross-sectional in nature, can provide strong evidence that smoking cessation can lead to regression of carotid atherosclerosis rather than being a result of a smaller number of pack-years of cigarettes smoked in former smokers.

We found only two studies describing the effects of cigarette smoking on IMT in Chinese populations. Thomas $\mathrm{et}^{\mathrm{al}} \mathrm{l}^{11}$ found in 616 healthy Chinese volunteers that ever smokers compared to never smokers had a thicker IMT and worse endothelial function. Their multiple linear regression analysis showed that, among others, male gender and pack-years of smoking were associated with carotid IMT thickness, although the smoking-

Table 2 Comparison of CCA-IMT by smoking status in men

\begin{tabular}{|c|c|c|c|c|c|}
\hline & \multirow[b]{2}{*}{$\begin{array}{l}\text { Never } \\
(n=375)\end{array}$} & \multirow[b]{2}{*}{$\begin{array}{l}\text { Former } \\
(n=246)\end{array}$} & \multicolumn{2}{|c|}{ Current (pack-years) } & \multirow[b]{2}{*}{$\mathbf{p}$} \\
\hline & & & $\begin{array}{l}1-39 \\
(n=245)\end{array}$ & $\begin{array}{l}40+ \\
(n=93)\end{array}$ & \\
\hline Left CCA-IMT, mean (SD), $\mu \mathrm{m}$ & 748.5 (159) & $813.2(187)$ & $794.7(175)$ & $808.7(186)$ & $<0.001$ \\
\hline Right CCA-IMT, mean (SD), $\mu \mathrm{m}$ & $749.9(154)$ & $782.4(174)$ & $768.8(161)$ & $782.6(174)$ & 0.07 \\
\hline $\begin{array}{l}\text { Mean of left and right CCA-IMT, } \\
\text { mean (SD), } \mu \mathrm{m}\end{array}$ & $749.2(134)$ & $797.8(154)$ & $781.2(144)$ & $795.6(156)$ & $<0.001$ \\
\hline Demographic* & $731.2(7.6)$ & $770.5(9.2)$ & $780.4(9.0)$ & $771.8(15.6)$ & $<0.001$ \\
\hline Demographic plus BMI* & $728.4(7.5)$ & $766.5(9.1)$ & $784.8(8.9)$ & $777.3(15.3)$ & $<0.001$ \\
\hline Demographic plus BP* & $725.6(7.6)$ & $766.5(9.1)$ & $780.6(8.9)$ & $775.2(15.4)$ & $<0.001$ \\
\hline \multicolumn{6}{|l|}{ Carotid plaque, n (\%) } \\
\hline 0 & $266(70.9)$ & $163(66.3)$ & $155(63.3)$ & $47(51.1)$ & 0.001 \\
\hline 1 & $81(21.6)$ & $53(21.5)$ & $61(24.9)$ & $23(25.0)$ & \\
\hline $2+$ & $28(7.5)$ & $30(12.2)$ & $29(11.8)$ & $22(23.9)$ & \\
\hline
\end{tabular}

${ }^{*}$ Mean of left and right CCA-IMT adjusted for demographic characteristics including age, education and physical activity; BMI; BP including systolic and diastolic blood pressure, mean (SEM).

BMI, body mass index; BP, blood pressure; CCA-IMT, common carotid artery intima-medial thickness. 
Table 3 ORs for CCA atherosclerosis and carotid plaques by smoking status in men

\begin{tabular}{|c|c|c|c|c|c|}
\hline & \multicolumn{4}{|c|}{ Smoking status } & \multirow[b]{3}{*}{ p for Trend } \\
\hline & \multirow[b]{2}{*}{ Never } & \multirow[b]{2}{*}{ Former } & \multicolumn{2}{|l|}{ Current (pack-years) } & \\
\hline & & & $1-39$ & $40+$ & \\
\hline No with CCA atherosclerosis (\%) & $139(37.1)$ & $103(41.9)$ & $102(41.6)$ & $52(56.5)$ & - \\
\hline Crude $\mathrm{OR}$ & 1.00 & $1.22(0.88$ to 1.70$)$ & $1.21(0.87$ to 1.68$)$ & $2.21(1.39 \text { to } 3.50)^{* * *}$ & 0.004 \\
\hline Model A I & 1.00 & 1.09 (0.77 to 1.54$)$ & $1.56(1.10 \text { to } 2.20)^{*}$ & $2.08(1.28 \text { to } 3.37)^{* *}$ & 0.001 \\
\hline \multicolumn{6}{|l|}{ Carotid plaque } \\
\hline No with carotid plaques (\%) & $109(29.1)$ & $83(33.7)$ & $90(36.7)$ & $45(48.9)$ & - \\
\hline Crude $\mathrm{OR}$ & 1.00 & 1.24 (0.88 to 1.76$)$ & $1.42(1.01 \text { to } 2.01)^{*}$ & $2.25(1.43 \text { to } 3.54)^{* * * *}$ & $<0.001$ \\
\hline Model A I & 1.00 & $1.14(0.80$ to 1.62$)$ & $1.71(1.20 \text { to } 2.44)^{* *}$ & $2.15(1.35 \text { to } 3.42)^{* * *}$ & $<0.001$ \\
\hline Model A II† & 1.00 & $1.13(0.78$ to 1.63$)$ & $1.81(1.25 \text { to } 2.64)^{* *}$ & $2.43(1.45 \text { to } 4.05)^{* * *}$ & $<0.001$ \\
\hline
\end{tabular}

Model A I: binary logistic regression model for CCA atherosclerosis (yes/no)/presence of carotid plaques (yes/no) with adjustment for age; model A II: binary logistic regression model for CCA atherosclerosis (yes/no)/presence of carotid plaques (yes/no) with adjustment for age, education, physical activity, body mass index, fasting triglyceride, low-density lipoprotein cholesterol, high-density lipoprotein cholesterol, total cholesterol and glucose, systolic and diastolic blood pressure; model B: ordinal logistic regression for CCA atherosclerosis grade/number of carotid plaques with adjustment for all factors above.

${ }^{*} \mathrm{p}<0.05 ;{ }^{* *} \mathrm{p}<0.01 ; * * * \mathrm{p}<0.001$.

†After excluding 27 participants with missing data on physical activity.

CCA, common carotid artery.

related IMT changes did not reach significance in male-only analyses, probably because their participants were younger and were apparently healthy. However, the more sensitive endothelial function measures were significantly worse in smokers, including in the male-only analyses. ${ }^{11}$ These detrimental effects were significant despite the use of "ever" smokers rather than separating current and former smokers. Pooling former smokers with current smokers into ever-smokers would under-estimate the OR. The other study of 1781 Taiwanese Chinese showed no significant association between smoking and carotid IMT and plaques, but the authors did not specify whether former smokers were classified as ever-smokers or non-smokers. ${ }^{21}$

Our results on the association between smoking and CCA atherosclerosis are consistent with most studies in Western populations, which have shown that cigarette smoking is associated with increased CCA-IMT. ${ }^{45} 1635{ }^{36}$ A prospective study

Table 4 ORs of CCA atherosclerosis and carotid plaque from smoking cessation in men

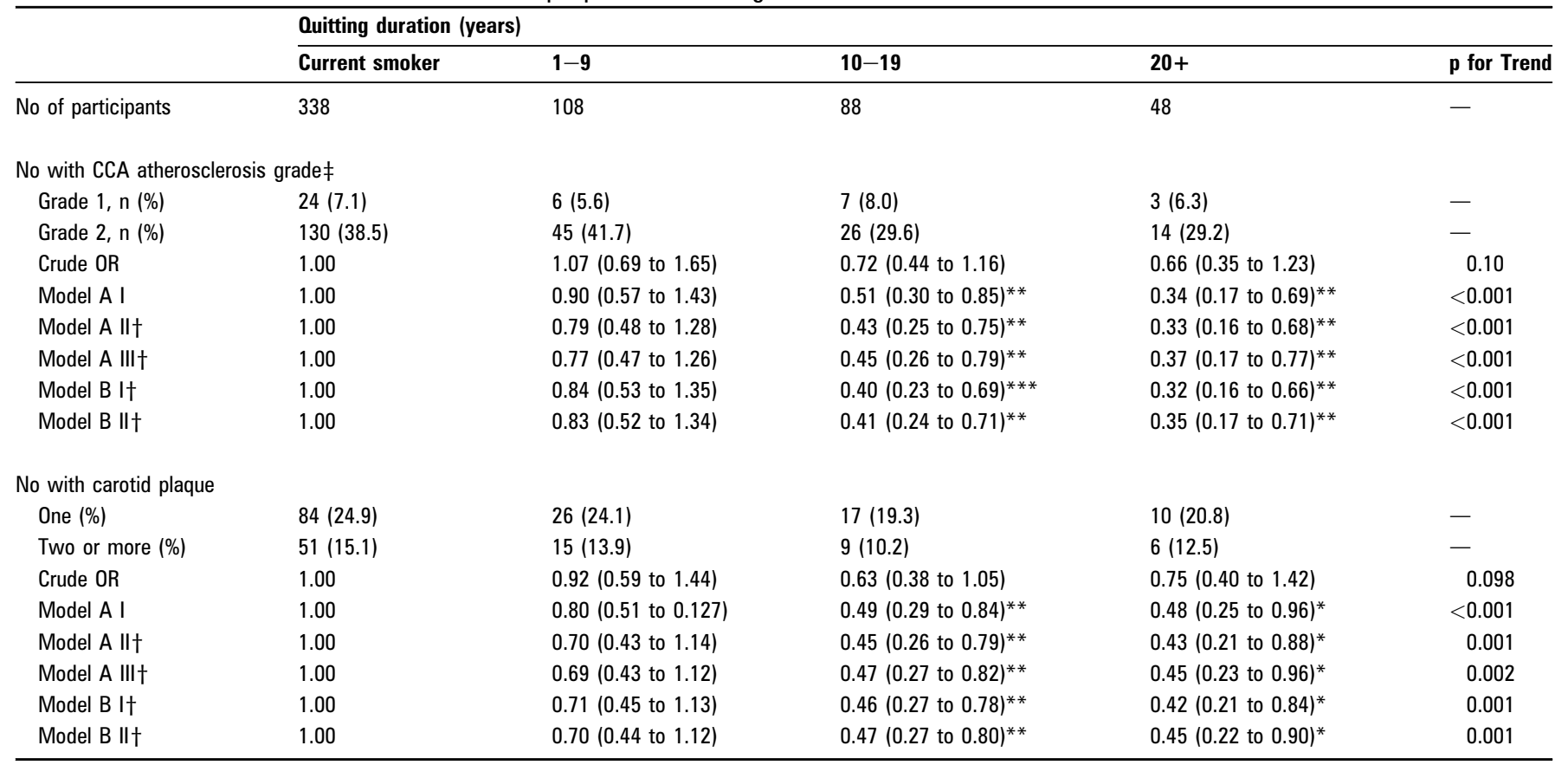

Model A I: binary logistic regression model for CCA atherosclerosis or presence of carotid plaques (yes/no) with adjustment for age; model A II: binary logistic regression model for CCA atherosclerosis or presence of carotid plaques (yes/no) with adjustment for age, education, physical activity, body mass index, fasting triglyceride, low-density lipoprotein cholesterol, highdensity lipoprotein cholesterol, total cholesterol and glucose, systolic and diastolic blood pressure; model A III: binary logistic regression model for CCA atherosclerosis or presence of carotid plaques (yes/no) with adjustment for all factors above plus pack-years; model B I: ordinal logistic regression for CCA atherosclerosis grade or number of carotid plaques (0, $1, \geq 2)$ with adjustment for all factors above; model B II: ordinal logistic regression for CCA atherosclerosis grade or number of carotid plaques $(0,1, \geq 2)$ with adjustment for all factors above plus packyears.

${ }^{*} \mathrm{p}<0.05 ;{ }^{* *} \mathrm{p}<0.01 ;{ }^{* * *} \mathrm{p}<0.001$.

†After excluding 27 participants with missing data on physical activity

$\neq$ Grade 0 for CCA-IMT $<1.0 \mathrm{~mm}$ or no observable CCA plaque; grade 1 for CCA-IMT $\geq 1.0 \mathrm{~mm}$ and $\leq 1.2 \mathrm{~mm}$; grade 2 for CCA-IMT $\geq 1.2 \mathrm{~mm}$ or with a diameter stenosis $\geq 20 \%$.

CCA, common carotid artery. 
by Diez-Roux et $a l^{18}$ with a follow-up of $12-14$ years also showed that passive and active smoking was associated with thickening of the CCA-IMT. However, none of the crosssectional or cohort studies described above examined for the association between quitting smoking and CCA atherosclerosis.

Studies investigating quitting are scarce with only one crosssectional ${ }^{16}$ and three prospective studies, but none showed risk reversal from quitting and all were of short follow-up duration. ${ }^{171920}$ A prospective study from the Netherlands found that 2 years of smoking cessation did not result in a slower progression or a regression in carotid IMT. ${ }^{19}$ However, given the underpowered nature of the study, which included only 33 quitters and had a short duration of smoking cessation, such observations are not surprising. Data from Fan's 3-year follow-up study of the Los Angeles Atherosclerosis Study of utility workers suggested a similar level of progression in former as current smokers, although male former smokers had lower basal carotid IMT levels. ${ }^{20}$ However, the difference between former and never smokers was inconsistent, with the text suggesting that the progression of thickening of the IMT in former smokers was greater than that in never smokers $(p=0.006$ for women, and $\mathrm{p}<0.0001$ for men), which is supported by their data that smokers progress more rapidly than never smokers. However, the tabulated data suggested no difference in progression between the groups. ${ }^{20}$ The Atherosclerosis Risk in Communities Study in the USA found that current smokers (50\%) and past smokers $(25 \%)$ showed more progression in carotid IMT than never smokers after a 3-year follow-up. However, after controlling for pack-years, no difference in the rate of progression of IMT thickening was found between past and current smokers. ${ }^{17}$ In contrast, our data clearly shows that the benefits of quitting with regards to CCA atherosclerosis improved with duration of abstinence. However, such risk reduction is only likely to be identified if the duration of quitting is over 10 years, which was not the case in any of the previous studies. ${ }^{16} 171920$ Given that CCA atherosclerosis is an independent predictor of vascular disease mortality, ${ }^{37}$ and the benefits of quitting smoking in reducing mortality is clear, ${ }^{38}$ our observations are likely to be valid.

\section{STRENGTHS AND LIMITATIONS}

Our study has several limitations. First, our cross-sectional results cannot provide definitive evidence for causation or risk reversal, but they are in accordance with prospective studies in Western populations that do provide causal evidence between smoking and quitting and cardiovascular disease. Prospective studies of smoking cessation in Chinese are rare, and we plan to follow-up our participants to longitudinally assess changes in atherosclerosis. Second, our sample size was not large enough so that we did not have sufficient number of ever smokers in women. Our results cannot be generalized to women, although there are no reasons to believe that smoking will not increase atherosclerosis in women. The small sample size also limited the precision of the risk estimates. Third, our participants are relatively healthy survivors. However, as smoking promotes progression, those who continue to smoke would have increasingly thicker CCA-IMT, even if quitting does not result in regression. As those with CCA atherosclerosis are more likely to die prematurely, the absence of a survivor effect would result in mean CCA-IMT or diameter stenosis levels being even greater, further emphasizing the benefits of quitting. Therefore, the risk estimates and benefits for quitters that we present are likely to be under-estimates. Finally, as smoking status was self-reported, we cannot rule out recall bias in these participants. However, as the participants did not know the study hypothesis or results of their ultrasound, and there was good

\section{What is already known on this subject}

Previous studies showed that cigarette smoking is an important risk factor for carotid atherosclerosis. Until now, evidence for the beneficial effect of quitting smoking on carotid atherosclerosis after removing the confounding effect of cigarette pack-year has been lacking, which was probably due to the short duration of cessation.

\section{What this study adds}

Smoking cessation is beneficial in attenuating the risk of carotid atherosclerosis associated with cigarette smoking. A longer duration of quitting smoking $(20+$ years) is significantly associated with decreasing the risk of the presence and severity of atherosclerosis.

reproducibility in self-reporting for this variable, strong recall bias is unlikely. Moreover, the diagnostic bias was unlikely as the physician performing the ultrasound measurements was blinded to the smoking status of the participants. Despite the crosssectional nature of the study, our results are consistent and coherent with previous studies in other populations and are biologically plausible, and support early smoking cessation, which can reverse the risks of many smoking-related diseases, probably including carotid atherosclerosis, in this population. Smoking cessation may be accompanied by other lifestyle changes. Although we have adjusted for lifestyle factors, further studies on the effects of other lifestyle changes and their interaction with quitting are warranted.

\section{CONCLUSIONS}

Smoking cessation is beneficial in attenuating the risk of carotid atherosclerosis associated with cigarette smoking. The short duration of cessation in earlier studies is a likely explanation for the inconsistent results.

Acknowledgements The Guangzhou Biobank Cohort Study-CVD investigators include: Guangzhou Number 12 Hospital: JM Lin, XJ Yue, CQ Jiang (co-PI); The University of Hong Kong: TH Lam; The Chinese University of Hong Kong: B Tomlinson, KS Wong; and The University of Birmingham: B Cheung, GN Thomas (co-PI).

Funding The study is funded by an NSFC/RGC (No 30518001; HKU720/05) grant; The University of Hong Kong Foundation for Educational Development and Research, Hong Kong; the Guangzhou Public Health Bureau and the Guangzhou Science and Technology Bureau, Guangzhou, China; and University of Birmingham, UK.

\section{Competing interests None.}

Patient consent Obtained.

Ethics approval The Guangzhou Medical Ethics Committee of the Chinese Medica Association approved the study, and all participants gave written, informed consent before participation.

Provenance and peer review Not commissioned; externally peer reviewed.

\section{REFERENCES}

1. Yang G, Fan L, Tan J, et al. Smoking in China: findings of the 1996 National Prevalence Survey. JAMA 1999;282:1247-53.

2. Sung HY, Wang L, Jin S, et al. Economic burden of smoking in China, 2000. Tob Control 2006;15(Suppl 1):i5-11.

3. Jiang CO, Lao XQ, Yin $\mathrm{P}$, et al. Smoking, smoking cessation and aortic arch calcification in older Chinese: the Guangzhou Biobank Cohort Study. Atherosclerosis 2009;202:529-34. 
4. Bonithon-Kopp C, Scarabin PY, Taquet A, et al. Risk factors for early carotid atherosclerosis in middle-aged French women. Arterioscler Thromb 1991;11:966-72.

5. Lorenz MW, Markus HS, Bots ML, et al. Prediction of clinical cardiovascular events with carotid intima-media thickness: a systematic review and meta-analysis. Circulation 2007:115:459-67.

6. Davis PH, Dawson JD, Riley WA, et al. Carotid intimal-medial thickness is related to cardiovascular risk factors measured from childhood through middle age: the Muscatine Study. Circulation 2001;104:2815-19.

7. Kawamoto R, Tomita H, Ohtsuka N, et al. Metabolic syndrome, diabetes and subclinical atherosclerosis as assessed by carotid intima-media thickness. J Atheroscler Thromb 2007;14:78-85.

8. Wildman RP, Schott LL, Brockwell S, et al. A dietary and exercise intervention slows menopause-associated progression of subclinical atherosclerosis as measured by intima-media thickness of the carotid arteries. J Am Coll Cardiol 2004:44:579-85.

9. Brohall G, Oden A, Fagerberg B. Carotid artery intima-media thickness in patients with type 2 diabetes mellitus and impaired glucose tolerance: a systematic review. Diabet Med 2006;23:609-16.

10. Baldassarre D, Amato $\mathrm{M}$, Bondioli $\mathrm{A}$, et al. Carotid artery intima-media thickness measured by ultrasonography in normal clinical practice correlates well with atherosclerosis risk factors. Stroke 2000;31:2426-30.

11. Thomas GN, Chook P, Yip TW, et al. Smoking without exception adversely affects vascular structure and function in apparently healthy Chinese: implications in global atherosclerosis prevention. Int J Cardiol 2008;128:172-7.

12. O'Leary DH, Polak JF, Kronmal RA, et al. Carotid-artery intima and media thickness as a risk factor for myocardial infarction and stroke in older adults. Cardiovascular Health Study Collaborative Research Group. N Engl J Med 1999;340:14-22.

13. Chien KL, Su TC, Jeng JS, et al. Carotid artery intima-media thickness, carotid plaque and coronary heart disease and stroke in Chinese. PLoS One 2008;3:e3435.

14. Chambless LE, Folsom AR, Clegg LX, et al. Carotid wall thickness is predictive of incident clinical stroke: the Atherosclerosis Risk in Communities (ARIC) study. Am J Epidemiol 2000;151:478-87.

15. Poredos $\mathbf{P}$, Orehek M, Tratnik E. Smoking is associated with dose-related increase of intima-media thickness and endothelial dysfunction. Angiology 1999;50:201-8

16. Howard G, Burke GL, Szklo M, et al. Active and passive smoking are associated with increased carotid wall thickness. The Atherosclerosis Risk in Communities Study. Arch Intern Med 1994;154:1277-82.

17. Howard G, Wagenknecht LE, Burke GL, et al. Cigarette smoking and progression of atherosclerosis: The Atherosclerosis Risk in Communities (ARIC) Study. JAMA 1998;279:119-24.

18. Diez-Roux AV, Nieto FJ, Comstock GW, et al. The relationship of active and passive smoking to carotid atherosclerosis 12-14 years later. Prev Med 1995;24:48-55.

19. van den Berkmortel $\mathbf{F W}$, Wollersheim $\mathrm{H}$, van Langen $\mathrm{H}$, et al. Two years of smoking cessation does not reduce arterial wall thickness and stiffness. Neth J Med 2004;62:235-41

20. Fan $\mathbf{A Z}$, Paul-Labrador M, Merz CN, et al. Smoking status and common carotid artery intima-medial thickness among middle-aged men and women based on ultrasound measurement: a cohort study. BMC Cardiovasc Disord 2006;6:42.
21. Sun Y, Lin CH, Lu CJ, et al. Carotid atherosclerosis, intima media thickness and risk factors - an analysis of 1781 asymptomatic subjects in Taiwan. Atherosclerosis 2002;164:89-94

22. Suurkula $\mathbf{M}$, Agewall $\mathrm{S}$, Fagerberg $B$, et al. Multiple risk intervention in high-risk hypertensive patients. A 3-year ultrasound study of intima-media thickness and plaques in the carotid artery. Risk Intervention Study (RIS) Group. Arterioscler Thromb Vasc Biol 1996;16:462-70.

23. Ornish D, Brown SE, Scherwitz LW, et al. Can lifestyle changes reverse coronary heart disease? The Lifestyle Heart Trial. Lancet 1990;336:129-33.

24. Kim SH, Lee SJ, Kang ES, et al. Effects of lifestyle modification on metabolic parameters and carotid intima-media thickness in patients with type 2 diabetes mellitus. Metabolism 2006;55:1053-9.

25. Markus RA, Mack WJ, Azen SP, et al. Influence of lifestyle modification on atherosclerotic progression determined by ultrasonographic change in the common carotid intima-media thickness. Am J Clin Nutr 1997;65:1000-4.

26. Anderssen SA, Hjelstuen AK, Hjermann I, et al. Fluvastatin and lifestyle modification for reduction of carotid intima-media thickness and left ventricular mass progression in drug-treated hypertensives. Atherosclerosis 2005;178:387-97.

27. Rabkin SW. Effect of cigarette smoking cessation on risk factors for coronary atherosclerosis. A control clinical trial. Atherosclerosis 1984;53:173-84.

28. Jiang CQ, Thomas GN, Lam TH, et al. Cohort profile: the Guangzhou Biobank Cohort Study, a Guangzhou-Hong Kong-Birmingham collaboration. Int J Epidemiol 2006; 35:844-52.

29. Jiang CQ, Lam TH, Lin JM, et al. An overview of the Guangzhou Biobank Cohort Study-Cardiovascular Disease Subcohort (GBCS-CVD): a platform for multidisciplinary collaboration. J Hum Hypertens 2009;24:139-50.

30. Deng HB, Macfarlane DJ, Thomas GN, et al. Reliability and validity of the IPAQ-Chinese: the Guangzhou Biobank Cohort study. Med Sci Sports Exerc 2008;40:303-7.

31. Thomas GN, Young RP, Tomlinson B, et al. A sibling-pair analysis of fasting lipids and anthropometric measurements and their relationship to hypertension. Clin Exp Hypertens 1999;21:1161-76.

32. Simon A, Gariepy J, Chironi G, et al. Intima-media thickness: a new tool for diagnosis and treatment of cardiovascular risk. J Hypertens 2002;20:159-69.

33. Zureik M, Ducimetiere P, Touboul PJ, et al. Common carotid intima-media thickness predicts occurrence of carotid atherosclerotic plaques: Iongitudinal results from the Aging Vascular Study (EVA) study. Arterioscler Thromb Vasc Biol 2000;20:1622-9.

34. Lam TH, He Y, Li LS, et al. Mortality attributable to cigarette smoking in China. JAMA 1997;278:1505-8.

35. van den Berkmortel FW, Smilde TJ, Wollersheim $\mathrm{H}$, et al. Intima-media thickness of peripheral arteries in asymptomatic cigarette smokers. Atherosclerosis 2000; 150:397-401.

36. Salonen R, Salonen JT. Determinants of carotid intima-media thickness: a populationbased ultrasonography study in eastern Finnish men. J Intern Med 1991;229:225-31.

37. Psaty BM, Furberg CD, Kuller LH, et al. Traditional risk factors and subclinical disease measures as predictors of first myocardial infarction in older adults: the Cardiovascular Health Study. Arch Intern Med 1999;159:1339-47.

38. Barzi F, Huxley R, Jamrozik K, et al. Association of smoking and smoking cessation with major causes of mortality in the Asia Pacific Region: the Asia Pacific Cohort Studies Collaboration. Tob Control 2008;17:166-72. 